\title{
Face Mask Detection and Sanitizer Despenser with Temperature Detection
}

\author{
Akash Thakre, Pravin Hande, Abhishek Pounikar, Jaydeo Dabre, Prof. Virendra Yadav
}

Computer Science and Engineering Department, Priyadarshini Institute of Engineering Technology, Nagpur, Maharashtra, India

\begin{abstract}
Article Info

Volume 8, Issue 3

Page Number : 509-515

\section{Publication Issue}

May-June-2021

\section{Article History}

Accepted : 01 June 2021

Published : 06 June 2021

In the present scenario due to Covid-19, the need for face mask detection applications, temperature detection and hand sanitizing are now in high demand for Railway Entrance, Airport Entrance, Office Entrance, Museums and Amusement Parks, Other Public Places and enterprises to ensure safety. These steps are now done in manual way by which the personnel may get in contact with the other personnel while sanitizing and checking temperature might not be accurate. To mitigate the problem, aiming to increase Covid-19 entrance safety, covering several relevant aspects: Contactless temperature sensing, Mask detection, Automatic hand sanitizing. Contactless temperature sensing subsystem relies on Raspberry Pi using temperature sensor,while mask detection performed by leveraging computer vision techniques on cameraequipped Raspberry $\mathrm{Pi}$, then the automatic hand sanitizing is achieved by the DC motor connected with the sensor and Raspberry Pi. Any person without temperature check, hand sanitizing and mask scan will not be provided entry. Only person having the conditions satisfied by the system is instantly allowed inside, else the buzzer will alert the security about the situation, if any violation of the condition is found. From the simulation results, it is clearly observed that the proposed method has high accuracy compare to the existing methods. Thus the system provides a 100\% automated system to prevent the spread of Covid-19.
\end{abstract}

Keywords : Contactless temperature sensing, Mask detection, Automatic hand sanitizing, Raspberry Pi, DCmotor, pi camera, Arm processor.

\section{INTRODUCTION}

The main aim of the work is to build a Raspberry pi based safety device for covid-19 safety rules to reduce the disease spread. We focus on most common indoor measurement system to allow the people. This work makes a use of MLX90614 contactless temperature sensor to detect the body temperature and pi camera, openCV to detect the mask whether the person is wearing a mask or not. We introduce an affordable COVID-19 indoor safety system. All modules and sensor are interfaced to the raspberry pi3 processor. 
The temperature sensor measures persons temperature using contactless IR sensor. The persons pass one by one. In case that persons temperature exceeds average human body, and then raspberry pi3 processor generates signal to lock the door and gives the audible alert through buzzer. Otherwise, the door is opened to let the person in.

For implementation of mask detection using an OpenCV and pi camera interfaced to the Raspberry Pi. When the user switches on the kit then pi camera capture the images, In case that image does not contain mouth and nose, it means that person wears mask properly and corresponding door will be opened. However, if the person not wear a mask then raspberry pi3 processor generates signal to lock the door and also gives the audible alerts through buzzer.

Sanitization: when the person place the hands in front of the ultrasonic sensor it will detects the hands and process this data to raspberry pi then raspberry pi spray the sanitizer automatically.

The main controlling device of the work is Raspberry pi3 processor. Here we are using DC motor as door. The SD card is a key part of the Raspberry $\mathrm{Pi}$; it provides the initial storage for the Operating System and files. The status of the work will be displays on LCD module. Here we are using two LEDs for working indication of the work.

\section{Literature Review}

In this section discuss the some of the recent techniques for face mask detection techniques using a Raspberry pi methods. proposed a system that restrict the growth of COVID-19 by finding out people who are not wearing any facial mask in a smart city network where all the public places are monitored with Closed-Circuit Television (CCTV) cameras. While a person without a mask is detected, the corresponding authority is informed through the city network. A deep learning architecture is trained on a dataset that consists of images of people with and without masks collected from various sources. The trained architecture achieved $98.7 \%$ accuracy on distinguishing people with and without a facial mask for previously unseen test data. It is hoped that our study would be a useful tool to reduce the spread of this communicable disease for many countries i aiming to help organizations respect the COVID-19 safety rules and guidelines in order to reduce the disease spread is presented. It focus on most common indoor measures people with high body temperature should stay at home, wearing mask is obligatory and distance between persons should be at 11 least 1.5-2 meters. For the first scenario, Arduino Uno microcontroller board with contactless temperature sensor is used, while we rely on Raspberry Pi singleboard computer equipped with camera making use of computer vision techniques for other two scenarios. Python version of OpenCV, open-source computer vision library was used for implementation of mask detection and social distance check algorithms. implemented a low-cost smart hand sanitizer dispenser with door controller based on Ultrasonic sensor that can help to solve the challenges faced by security guards at different stations such as bank doors, school gates, hospital gates etc. in enforcing this hand sanitizing action before letting people in to where ever they intend to enter. That is to say, when a person(s) wants to access the entrance door, they must first sanitizer their hands or else the door will remain locked. With this smart hand sanitizer dispenser, an ultrasonic sensor is used to check the presence of hands below the outlet of the sanitizer machine. It will continuously calculate the distance between the sanitizer outlet and itself and tells the microcontroller to turn on the servo motor whenever the distance is less than $10 \mathrm{~cm}$ to push the sanitizer out and immediately after the sanitizer outlet dropping some amount into your hands, the electromagnetic lock will de-energize (unlock the door) lighting up a green LED and display a word "The Entrance Door is Open" on the LCD display, 
then the second servo motor will open the entrance door. Otherwise, the door will neither unlock nor open but a red LED will continuously be on with the "Please Sanitize Here" words displayed on the LCD display. From the above analysis it is observed that these methods have some limitations. It is a manual process which requires the man power. The person has to be trained to handle the temperature checking. Human error may happen. Scanning is skipped whenever no one is watching. Not suitable to handle large crowd. $\mathrm{n}$ the world.

\section{Problem Statement}

Recently India along with almost all big and small countries stated emergency conditions for the novel coronavirus (COVID-19). Practically, the whole population of the world is under lockdown and people are wearing a mask and hands sanitization as suggested by the World Health Organization (WHO). Precaution is always better than cure. But since there isn't any cure yet available, the only option we are left with is to follow the precautions. And failing to do so may has severe consequences.

Since there is no vaccine yet available in the market, the only way to be safe is by taking precautions. It is suggested to wear a face mask, that masks help in slowing down the spread of infection as the virus mainly gets transmitted with the aerosols which come out of an infected person's nose or mouth while coughing or sneezing. Currently, Temperature Checkups are done manually using Contactless Thermometer. Manual Checkups can be Inefficient, Impractical, and Risky.

To solve these problems, we have designed a raspberry pi based contact less body temperature measurement and face mask detector which detects whether the person is wearing a mask or not. Also automatic sanitizer to sanitize our hands. We focus on most common indoor measurement system to allow the people.

\section{PROPOSED METHODOLOGY}

In this work introduced a automated system for covering several relevant aspects like Contactless temperature sensing, Mask detection, Automatic hand sanitizing aiming to increase Covid-19 entrance safety. It is a purely automated system. No one is allowed to enter if they didn't wear mask and has high temperature. Buzzer will alert the security of this situation if any fail-safe condition is found. Additionally, if they have high temperature the message will be sent to the Covid19 health center. Initially sensor identify the person by the motion. Using temperature sensor the temperature of the person is detected and if it exceeds temperature limit a alert message is send.

Parallelly the camera captures the image. The image processed using Viola Jones technique using OpenCVand tensor flow, the classification between the person wearing mask or not is verified. The proximity sensor detects the hand of a person places his/her hand under the sanitizer and it dispenses the sanitizer accordingly. Each process of the proposed work is explained in briefly in the following section.

\section{A. PERSON DETECTION}

Person detection is the process of identifying a human's presence at the entrance. It is achieved using the Passive Infra Red sensor(PIR). When a person is detected the PIR sensor gives the output as 1 . When a person is not detected it gives the output as 0 .

\section{B. TEMPERATURE CHECK}

When a person is detected then the temperature of the person is checked using mlx90614 sensor. If the temperature is above $1000 \mathrm{~F}$ then an alert message is sent to the COVID centre. If the temperature is below $1000 \mathrm{~F}$ then the person is checked for face mask.

\section{FACE MASK DETECTION}

When the temperature is below $1000 \mathrm{~F}$ the facemask is detected using camera's input. For checking the facemask of a person, we use deep learning with 
image processing using python. The code is flashed inside the Raspberry Pi module.

\section{ALERTING SYSTEM}

If the facemask is not worn by the person then the buzzer supply is turned on by the Raspberry Pi. The buzzer will ring until the facemask is worn.

\section{REQUIREMENTS}

\section{RASPBERRY PI3 BOARD}

The Raspberry Pi is a credit-card-sized single-board computer developed in the UK by the Raspberry Pi Foundation with the intention of promoting the teaching of basic computer science in schools.

The Raspberry $\mathrm{Pi}$ is manufactured through licensed manufacturing deals with Newark element14 (Premier Farnell), RS Components and Egoman. All of these companies sell the Raspberry $\mathrm{Pi}$ online. Egoman produces a version for distribution solely in China and Taiwan, which can be distinguished from other Pi by their red coloring and lack of FCC/CE marks. The hardware is the same across all manufacturers.

\section{RASPBERRY PI 3 BOARD}

Silicon Manufacturer: Broadcom

Core Architecture: ARM

Core Sub-Architecture: ARM1176JZF-S

Silicon Core Number: BCM2837

Silicon Family Name: BCM2xxx

No. of Bits: 64 bit

Kit Contents: Raspberry Pi 3

Features: Credit card size computer, HDMI, Ethernet \& 4 USB ports, 1GB RAM, Micro SD Socket, 40 GPIO

\section{ARM PROCESSOR}

ARM Processor was developed at Acorn computer limited of Cambridge, England between 1983 and 1985.

This was after RISC concept came out at Stanford and Berkeley universities in 1980.

ARM uses Enhanced RISC Architecture.

ARM (Acorn RISC machine) limited was found in 1990.

ARM designed basic core structure and licensed it to many partners who develop and fabricate new Micro Controllers and different chips.

ARM processor is mainly intended in the development of embedded applications which involve complex computations (High-end applications).

\section{Adapter (Power supply)}

The $\mathrm{AC}$ adapter, $\mathrm{AC} / \mathrm{DC}$ adapter or $\mathrm{AC} / \mathrm{DC}$ converter is a type of external power supply, often enclosed in a case similar to an AC plug. Other names include plug pack, plug-in adapter, adapter block, domestic mains adapter, line power adapter, wall wart, or power adapter. AC adapters are used with electrical devices that require power but do not contain internal components to derive the required voltage and power from mains power. The internal circuitry of an external power supply is very similar to the design that would be used for a built-in or internal supply.

External power supplies are used both with equipment with no other source of power and with battery-powered equipment, where the supply, when plugged in, can sometimes charge the battery in addition to powering the equipment.

Use of an external power supply allows portability of battery-powered equipment without the added bulk of internal power components and makes it unnecessary to produce equipment for use only with a specified power source. 


\section{LED}

A light-emitting diode (LED) is a semiconductor light source. LEDs are used as indicator lamps in many devices, and are increasingly used for lighting. Introduced as a practical electronic component in 1962, early LEDs emitted low-intensity red light, but modern versions are available across the visible, ultraviolet and infrared wavelengths, with very high brightness.

\section{MLX90614(Temperature sensor)}

The MLX90614 is a Contactless Infrared (IR) Digital Temperature Sensor that can be used to measure the temperature of a particular object ranging from $-70^{\circ} \mathrm{C}$ to $382.2^{\circ} \mathrm{C}$. The sensor uses IR rays to measure the temperature of the object without any physical contact and communicates to the microcontroller using the I2C protocol.

\section{6. pi Camera}

The Camera Module can be used to take highdefinition video, as well as stills photographs. ... It supports 1080p30, 720p60 and VGA90 video modes, as well as still capture. It attaches via a $15 \mathrm{~cm}$ ribbon cable to the CSI port on the Raspberry Pi.

The camera consists of a small $(25 \mathrm{~mm}$ by $20 \mathrm{~mm}$ by $9 \mathrm{~mm}$ ) circuit board, which connects to the Raspberry Pi's Camera Serial Interface (CSI) bus connector via a flexible ribbon cable. The camera's image sensor has a native resolution of five megapixels and has a fixed focus lens. The software for the camera supports full resolution still images up to $2592 \times 1944$ and video resolutions of 1080p30, 720p60 and 640x480p60/90

\section{BUZZER}

Basically, the sound source of a piezoelectric sound component is a piezoelectric diaphragm. A piezoelectric diaphragm consists of a piezoelectric ceramic plate which has electrodes on both sides and a metal plate (brass or stainless steel, etc.). A piezoelectric ceramic plate is attached to a metal plate with adhesives. Applying D.C. voltage between electrodes of a piezoelectric diaphragm causes mechanical distortion due to the piezoelectric effect. For a misshaped piezoelectric element, the distortion of the piezoelectric element expands in a radial direction. And the piezoelectric diaphragm bends toward the direction. The metal plate bonded to the piezoelectric element does not expand. Conversely, when the piezoelectric element shrinks, the piezoelectric diaphragm bends in the direction Thus, when AC voltage is applied across electrodes, the bending is repeated, producing sound waves in the air.

To interface a buzzer the standard transistor interfacing circuit is used. Note that if a different power supply is used for the buzzer, the 0V rails of each power supply must be connected to provide a common reference.

If a battery is used as the power supply, it is worth remembering that piezo sounders

draw much less current than buzzers. Buzzers also just have one tone, whereas a

piezo sounder is able to create sounds of many different tones.

To switch on buzzer -high 1

To switch off buzzer -low 1

\section{LCD DISPLAY:}

\section{LCD Background:}

One of the most common devices attached to a micro controller is an LCD display. Some of the most common LCDs connected to the many microcontrollers are $16 \times 2$ and 20x2 displays. This means 16 characters per line by 2 lines and 20 characters per line by 2 lines, respectively.

\section{DC. Motor:}

A dc motor uses electrical energy to produce mechanical energy, very typically through the interaction of magnetic fields and current- 
carrying conductors. The reverse process, producing electrical energy from mechanical energy, is accomplished by an alternator, generator or dynamo. Many types of electric motors can be run as generators, and vice versa. The input of a DC motor is current/voltage and its output is torque.

The DC motor has two basic parts: the rotating part that is called the armature and the stationary part that includes coils of wire called the field coils. The stationary part is also called the stator. Figure shows a picture of a typical DC motor, Figure shows a picture of a DC armature, and Fig shows a picture of a typical stator. From the picture you can see the armature is made of coils of wire wrapped around the core, and the core has an extended shaft that rotates on bearings. You should also notice that the ends of each coil of wire on the armature are terminated at one end of the armature. The termination points are called the commutator, and this is where the brushes make electrical contact to bring electrical current from the stationary part to the rotating part of the machine.

\section{Ultrasonic sensor:}

Ultrasonic sensors (also known as transceivers when they both send and receive, but more generally called transducers) work on a principle similar to radar or sonar which evaluates attributes of a target by interpreting the echoes from radio or sound waves respectively. Ultrasonic sensors generate high frequency sound waves and evaluate the echo which is received back by the sensor. Sensors calculate the time interval between sending the signal and receiving the echo to determine the distance to an object.

This technology can be used for measuring: wind speed and direction (anemometer), tank or channel level, and speed through air or water. For measuring speed or direction a device uses multiple detectors and calculates the speed from the relative distances to particulates in the air or water. To measure tank or channel level, the sensor measures the distance to the surface of the fluid. Further applications include: humidifiers, sonar, medical ultrasonography, burglar alarms and non-destructive testing.

Systems typically use a transducer which generates sound waves in the ultrasonic range, above 18,000 hertz, by turning electrical energy into sound, then upon receiving the echo turn the sound waves into electrical energy which can be measured and displayed.

The technology is limited by the shapes of surfaces and the density or consistency of the material. Foam, in particular, can distort surface level readings.

\section{Sanitizer:}

Although-alcohol-based hand sanitizers can quickly reduce the number of germs in many situations, they should be used in the right situations.

\section{RESULT}

The work Temperature and Mask Scan Entry System was designed an indoor measurement system to allow the people using some covid rules as body temperature ,automatic hand sanitizer and face mask detection. Raspberry pi3 based contact less body temperature measurement and face mask detector which detects whether the person is wearing a mask or not using Pi camera and OpenCV.

\section{CONCLUSION}

Integrating features of all the hardware components used have been developed in it. Presence of every module has been reasoned out and placed carefully, thus contributing to the best working of the unit. Secondly, using highly advanced ICs with the help of growing technology, the work has been successfully implemented. Thus the work has been successfully designed and tested. 


\section{FUTURE SCOPE}

WE can extend this work by adding some health sensors such as heartbeat, oxygen, respiration and IOT technology. The systems can include monitoring sensor data in IOT platform via wireless, the system can display sensor parameter on LCD. The system workout on COVID-19 to identify the number of COVID-19 cases in a region then we can classified as either red, orange or green zone. Moreover, timely and effectively by using this work we can managing the COVID-19 outbreak and reducing viral transmission.

\section{REFERENCES}

[1]. Coronavirus Update (Live) online]. Available on:

https://www.worldometers.info/coronavirus/, last accessed: 11/07/2020.

[2]. P. Zhai et al., The epidemiology, diagnosis and treatment of COVID-19, International Journal of Antimicrobial Agents vol. 55 issue 5, May 2020, 105955, pp. 1-13, 2020. https://doi.org/10.1016/j.ijantimicag.2020.10595 5

[3]. P. Dawson et al., Loss of Taste and Smell as Distinguishing Symptoms of COVID-19, Clinical Infectious Diseases June 2020, pp. 1-4, 2020. https://doi.org/10.1093/cid/ciaa799

[4]. L. Morawska, How can airborne transmission of COVID-19 indoors be minimised?, Environment International vol. 142, September 2020, 105832, pp. 1-7, 2020. https://doi.org/10.1016/j.envint.2020.105832

[5]. T. Galbadage, B. Peterson, R. Gunasekera, Does COVID-19 Spread Through Droplets Alone?, Frontiers in Public Health, vol. 8, April 2020, pp. $\quad 1-4, \quad 2020$. https://doi.org/10.3389/fpubh.2020.00163

[6]. Coronavirus Incubation Period online]. Available https://www.worldometers.info/coronavirus/co ronavirus-incubationperiod/ , last accessed: 11/07/2020.

[7]. D. Oran, E. Topol, Prevalence of Asymptomatic SARS-CoV-2 Infection: A Narrative Review, Annals of Internal Medicine, June 2020, pp. 17, 2020. https://doi.org/10.7326/M20-3012

[8]. T. Dbouk, D. Drikakis, On respiratory droplets and face masks, Physics of Fluids 32, 063303, pp. $1-11,2020$. https://doi.org/10.1063/5.0015044

[9]. Y. Song et al., COVID-19 Treatment: Close to a Cure? A Rapid Review of Pharmacotherapies for the Novel Coronavirus preprint], pp. 1-25, 2020.

https://doi.org/10.20944/preprints202003.0378. v1

[10]. V. Balachandar et al., COVID-19: emerging protective measures, European Review for Medical and Pharmacological Sciences vol. 24 (6), pp. 3422-3425, 2020.

\section{Cite this article as :}

Akash Thakre, Pravin Hande, Abhishek Pounikar, Jaydeo Dabre, Prof. Virendra Yadav, "Face Mask Detection and Sanitizer Despenser with Temperature Detection", International Journal of Scientific Research in Science and Technology (IJSRST), Online ISSN : 2395-602X, Print ISSN : 2395-6011, Volume 8 Issue 3, pp. 509-515, May-June 2021. Available at doi : https://doi.org/10.32628/IJSRST218391

Journal URL : https://ijsrst.com/IJSRST218391 